\title{
Fibrilação Atrial (Parte 2) - Ablação por Cateter
}

\author{
Atrial Fibrillation (Part 2) - Catheter Ablation

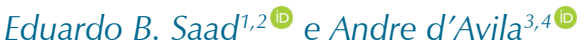 \\ Hospital Pró-Cardíaco - Serviço de Arritmias e Estimulação Cardíaca Artificial, ${ }^{1}$ Rio de Janeiro, RJ - Brasil \\ Hospital Samaritano, ${ }^{2}$ Rio de Janeiro, RJ - Brasil \\ Hospital SOS Cardio, ${ }^{3}$ Florianópolis, SC - Brasil \\ Beth Israel Deaconess Hospital, Harvard Medical School, ${ }^{4}$ Boston - EUA
}

\section{Resumo}

Após mais de 20 anos desde sua utilização inicial, a ablação por cateter se tornou um procedimento rotineiramente realizado para tratamento de pacientes com fibrilação atrial (FA). Fundamentado inicialmente no isolamento elétrico das veias pulmonares em pacientes com FA paroxística, subsequentes avanços no entendimento da fisiopatologia levaram a técnicas adicionais não só para obter melhores resultados, mas também para tratar pacientes com formas persistentes de arritmia, assim como pacientes com cardiopatia estrutural e insuficiência cardíaca.

Significativos avanços tecnológicos, em especial no mapeamento eletroanatômico 3D, no uso de ecocardiograma intracardíaco e na forma de energia aplicada (crioablação e força de contato tecidual com radiofrequência), permitiram redução significativa na taxa de complicações e no uso de radiação ionizante.

Atualmente, a ablação é o tratamento mais eficiente para pacientes com FA, sendo uma excelente alternativa ao uso de fármacos antiarrítmicos, cujo desenvolvimento foi insignificante nas últimas décadas.

Com as pioneiras observações feitas por Haissaguerre et al., ${ }^{1}$ demostrou-se o papel fundamental de focos arritmogênicos nas veias pulmonares (VP) na fisiopatologia da iniciação e na manutenção dos episódios de FA. Nestas foi estabelecido o conceito de FA focal, em que uma arritmia atrial que acomete difusamente ambos os átrios tem origem bem determinada, logo, passível de intervenções terapêuticas. Técnicas utilizando a ablação por cateter foram desenvolvidas e aperfeiçoadas, visando à eliminação dos focos geradores da FA por meio da ablação circunferencial ao redor das VP, ${ }^{2-4}$ com índices de sucesso e performance superiores quando comparados com a melhor terapêutica farmacológica. ${ }^{5-10}$

O objetivo deste artigo é revisar os avanços ocorridos no tratamento ablativo da FA e descrever para o cardiologista

\section{Palavras-chave}

Arritmias Cardíacas; Fibrilação Atrial/terapia; Ablação por Cateter/métodos; Ecocardiografia/métodos.

Correspondência: Eduardo B. Saad •

Rua Visconde de Pirajá 351, sala 623. CEP 22410-906, Rio de Janeiro, RJ - Brasil

E-mail: eduardobsaad@hotmail.com

Artigo recebido em 30/05/2020, revisado em 05/09/2020, aceito em $22 / 10 / 2020$

DOI: https://doi.org/10.36660/abc.20200477 clínico o atual estado da arte em relação a suas indicações, técnicas, resultados e complicações.

\section{Estratégias de ablação}

Ao longo dos últimos 20 anos, diversas estratégias de ablação foram utilizadas para controle da FA. Em comum, é consenso atual que o isolamento de todas as VPé fundamental em todos os grupos de pacientes (FA paroxística, persistente ou persistente de longa duração). ${ }^{11-15}$ Esse isolamento deve ser comprovado eletricamente por mapeamento circular no interior das VP (Figuras 1 e 2), pois essa etapa é primordial para o sucesso do procedimento. Estudos recentes mostram que deve, inclusive, ser realizado sem interrupção da anticoagulação oral, estratégia que reduz os riscos trombóticos e hemorrágicos. ${ }^{16-18}$

Em pacientes com FA paroxística, em geral, o isolamento das VP é tudo que é necessário, realizando-se lesões adicionais apenas em situações específicas (p. ex., focos deflagradores mapeados fora das VP). Alguns centros realizam de rotina o isolamento da veia cava superior, ${ }^{19,20}$ visto que também pode ser, mais raramente, fonte deflagradora de ectopias e arritmias, podendo induzir FA. A maioria das publicações demonstra resultados favoráveis, com taxas de sucesso superiores a 70\%.6,7,9

O isolamento das VP pode ser realizado utilizando: 1) energia de radiofrequência (RF), por meio de aplicações focais ponto a ponto (Figura $1 \mathrm{~A}$ ), idealmente com cateteres com sensores da pressão tecidual exercida (Figura 1 B), ou 2) congelamento (crioablação), utilizando um cateter-balão posicionado no antro das VP, capaz de realizar toda a lesão tecidual simultaneamente na circunferência em contato com o tecido (Figura 1D). Estudo randomizado (Fire and ICE ${ }^{21}$ comparando diretamente as duas estratégias para tratamento de FA paroxística demonstrou resultados semelhantes. Esses achados foram replicados em um segundo estudo randomizado (CIRCA-DOSE) ${ }^{22}$ que comparou dois regimes de crioablação (4min vs. 2 min de congelamento) ao uso da RF com força de contato para isolamento das VP em pacientes com FA paroxística. Nesse estudo, observou-se redução da carga de FA $>98 \%$ por meio de monitoramento eletrocardiográfico contínuo. Importante salientar que o cateterbalão usualmente não é utilizado para ablação em outras regiões além das VP; quando necessário, deve-se utilizar um cateter de RF (Figura 1C).

Já nas formas persistente e persistente de longa duração, a criação de barreiras elétricas adicionas é frequentemente realizada, visto que o simples isolamento das VP é insuficiente e associado a altas taxas de recorrência. ${ }^{23-25}$ Diversas estratégias foram estudadas, ${ }^{26-37}$ sendo as mais frequentemente utilizadas: ablação de deflagradores fora 

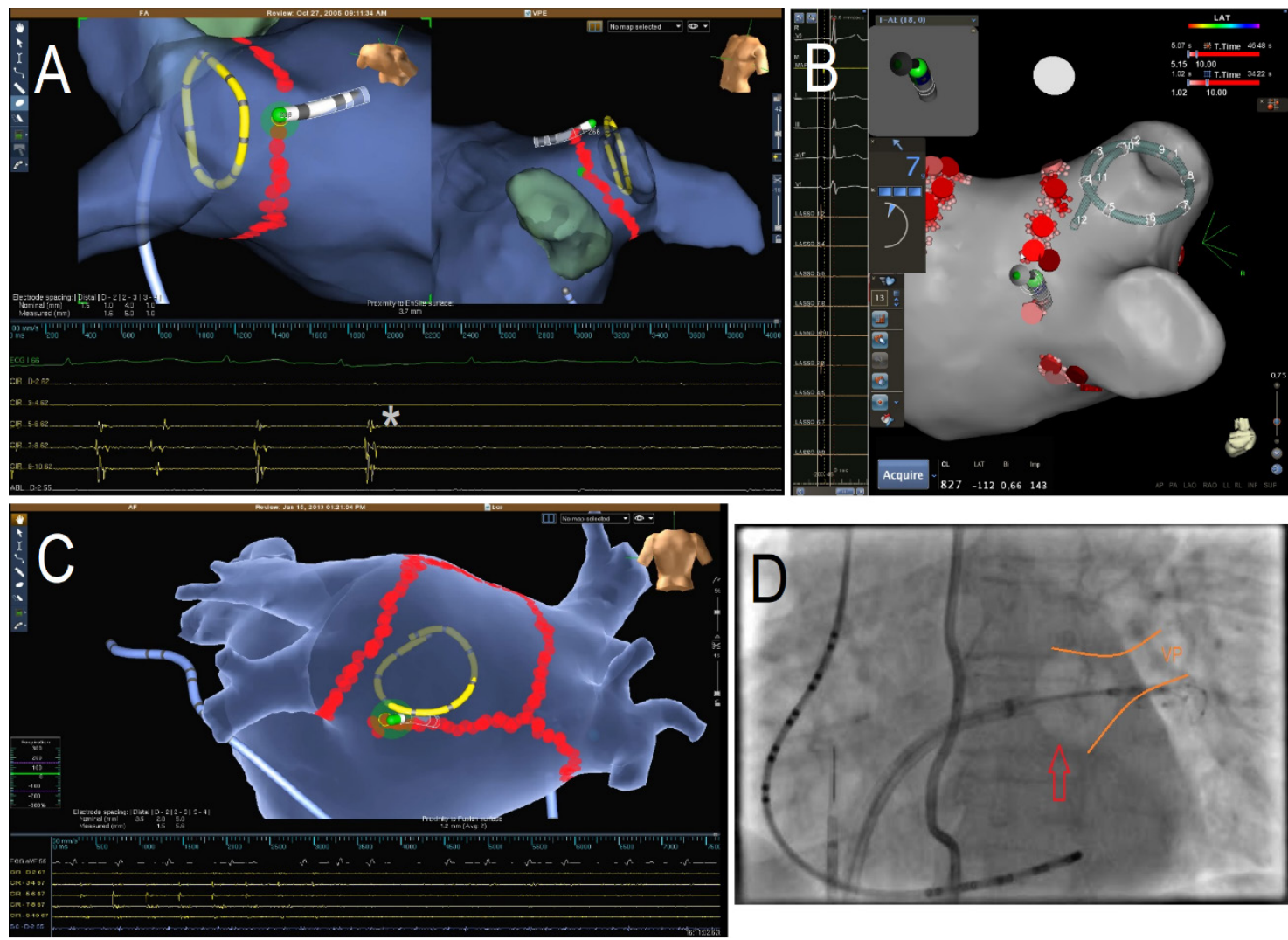

Figura 1 - Ablação por cateter para tratamento da FA paroxistica. A) Isolamento das VP esquerdas por ablação circunferencial (RF ponto a ponto) guiado por mapeamento eletroanatômico 3D (sistema NAVX - Abbott), demonstrando o desaparecimento dos eletrogramas $\left(^{*}\right)$ registrados por um cateter circular no interior das VP. B) Isolamento das VP direitas (sistema CARTO - Biosense Webster) com cateter com sensor de contato tecidual (demonstrado pelo vetor de força e pela quantificação = 7g); o cateter circular de mapeamento está no interior da VP superior direita. C) Ablação de FA persistente (sistema NAVx - Abbott) demonstrando a extensão das lesões de RF para isolamento da parede posterior do AE (lesão linear no teto e na parte inferior), levando ao desaparecimento dos eletrogramas na parede posterior (registrados pelo cateter circular de mapeamento). D) Imagem fluoroscópica durante crioablação para isolamento da VP superior esquerda, demonstrando o cateter-balão (seta) insuflado e em contato com o óstio dessa veia. A ablação em toda a circunferência da VP é realizada simultaneamente pelo balão, que usualmente é restrito para isolamento das VP - quando necessário ablação adicional, um cateter de RF deve ser utilizado.

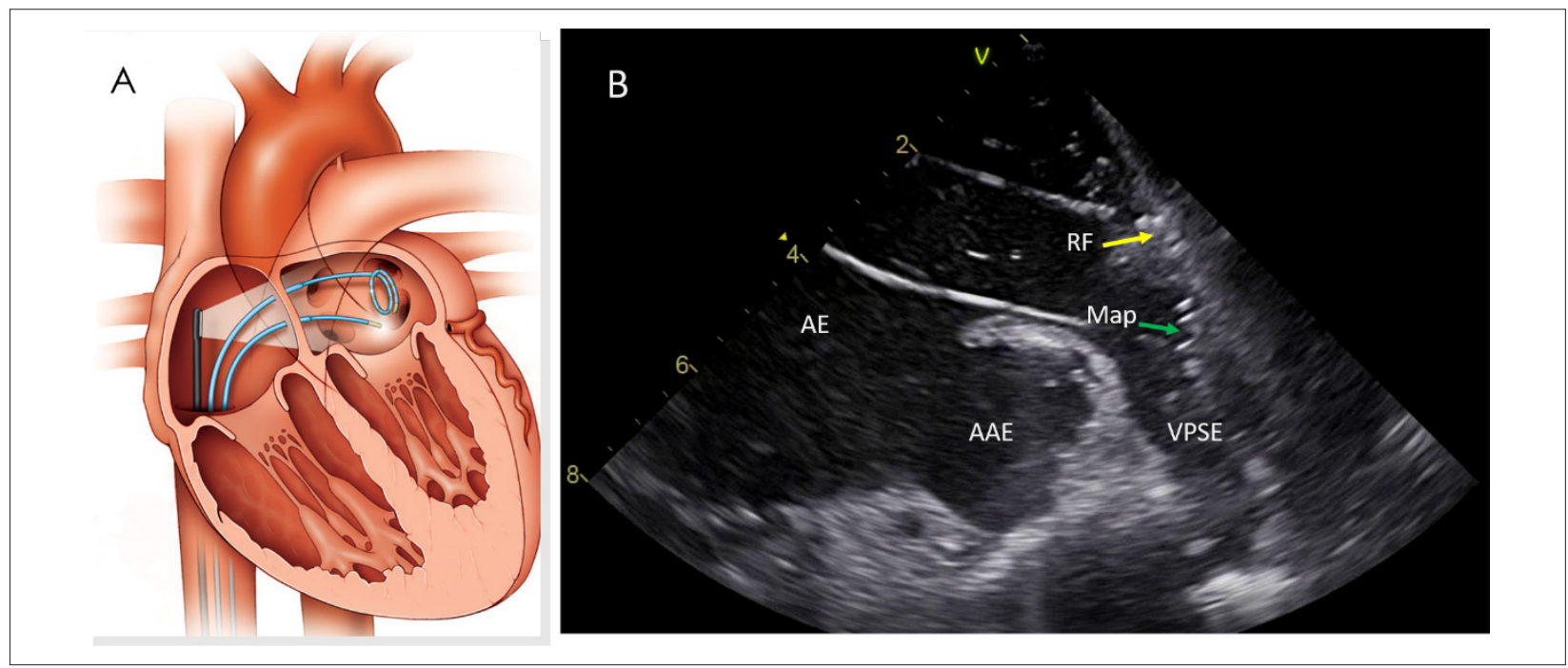

Figura 2 - Uso do eco intracardíaco (ICE) durante ablação da FA. A) Desenho esquemático mostrando o cateter de ICE na cavidade atrial direita com feixe direcionado para guiar as duas punções transeptais e o posicionamento dos cateteres de mapeamento circular e de ablação no AE. B) Imagem do ICE demonstrando o posicionamento antral e o contato tecidual durante aplicação de RF ao redor da VP superior esquerda (VPSE). AE: átrio esquerdo; AAE: apêndice atrial esquerdo; Map: cateter de mapeamento; RF: cateter de ablação. 
das veias pulmonares, lesões lineares no átrio esquerdo (AE) e extensas aplicações de RF em sítios com registro de eletrogramas fracionados durante a FA (mais comumente observados na parede posterior, septo, teto, anel mitral, base do apêndice atrial esquerdo (AAE) e no interior do seio coronário). Durante a aplicação de RF nesses locais, pode ocorrer reversão a taquicardias atriais regulares ou até mesmo ao ritmo sinusal.

Merece ressalva o resultado negativo do estudo randomizado Star AF II, ${ }^{38}$ que comparou a adição de lesões lineares e ablação de potenciais fragmentados ao isolamento das VP em pacientes com FA persistente. Nesse estudo, não houve diferença na manutenção do ritmo sinusal aos 18 meses entre os grupos (59\% para o isolamento apenas vs. 49\% e $46 \%$ nos outros grupos, sem significância estatística). Por isso, muitos centros ainda praticam apenas o isolamento das VP mesmo em pacientes com FA persistente.

Uma estratégia mais agressiva para eliminação de deflagradores foi também testada em um estudo randomizado controlado (BELIEF Trial) ${ }^{39}$ - o isolamento elétrico do AAE. Neste, o isolamento dessa estrutura em adição à ablação convencional foi associado à redução de $55 \%$ no risco relativo de recorrência de FA em uma população de pacientes com FA persistente de longa duração. O isolamento do AAE é atualmente realizado seletivamente devido ao fato de exigir extensas aplicações de RF e adicionar risco para fenômenos embólicos - a perda da contração do AAE leva à remora e formação de trombo. Pacientes com AAE eletricamente isolado devem ser anticoagulados cronicamente independente do escore CHADSVASC, e devem ser submetidos à oclusão dessa estrutura caso o anticoagulante seja contraindicado. ${ }^{40}$

Portanto, em formas mais persistentes de FA com significativo remodelamento atrial, há necessidade de mudança do substrato atrial, implicando maior número e extensão das aplicações de RF. Não há consenso na literatura quanto a melhor estratégia a ser utilizada (Tabela 1). A evolução da FA para formas persistentes representa progressão de um processo patológico ${ }^{41,42}$ e deve servir de motivação para o tratamento mais precoce, quando ainda paroxística. Um grande estudo retrospectivo com mais de 4.500 pacientes analisou o impacto do tempo entre o diagnóstico de FA e a realização da ablação. ${ }^{43}$ Os resultados são contundentes, demonstrando que quanto mais cedo a ablação é realizada, melhores são os resultados - estabelecendo o "conceito oncológico da FA", ou seja, os melhores resultados são obtidos quando se faz o tratamento nas fases iniciais da doença (isolamento das VP em pacientes com FA paroxística). Em fases mais avançadas (FA persistente e permanente), o tratamento é bem mais extenso e com resultados inferiores. Por isso, aqui também, quanto mais cedo, melhor.

\section{Tecnologias para guiar a ablação}

Independentemente da estratégia utilizada, métodos de mapeamento por imagem frequentemente são utilizados em adição ao tradicional mapeamento eletrofisiológico. Dois tipos de tecnologia são apropriados nesta circunstância:

a) Mapeamento eletroanatômico: esta forma de mapeamento em 3D permite definir com precisão a anatomia da cavidade atrial esquerda e das VP, delinear o substrato funcional pela medida da voltagem tecidual, marcar as lesões de RF (Figura 1) no mapa formado e traduzir em cores a informação elétrica obtida. É possível, também, navegar em imagens da anatomia real originada em tomografia computadorizada ou ressonância magnética. Essa metodologia é especialmente útil para reduzir o tempo de exposição à fluoroscopia, tornando de fácil apreciação o circuito ou o foco da arritmia e as lesões realizadas para tratá-las. Dois sistemas estão atualmente disponíveis no Brasil: CARTO - Biosense Webster e NavX - Abbott Medical.

b) Ecocardiograma intracardíaco (ICE): através de um cateter inicialmente posicionado no átrio direito, é possível obter

Tabela 1 - Estratégias durante ablação da fibrilação atrial

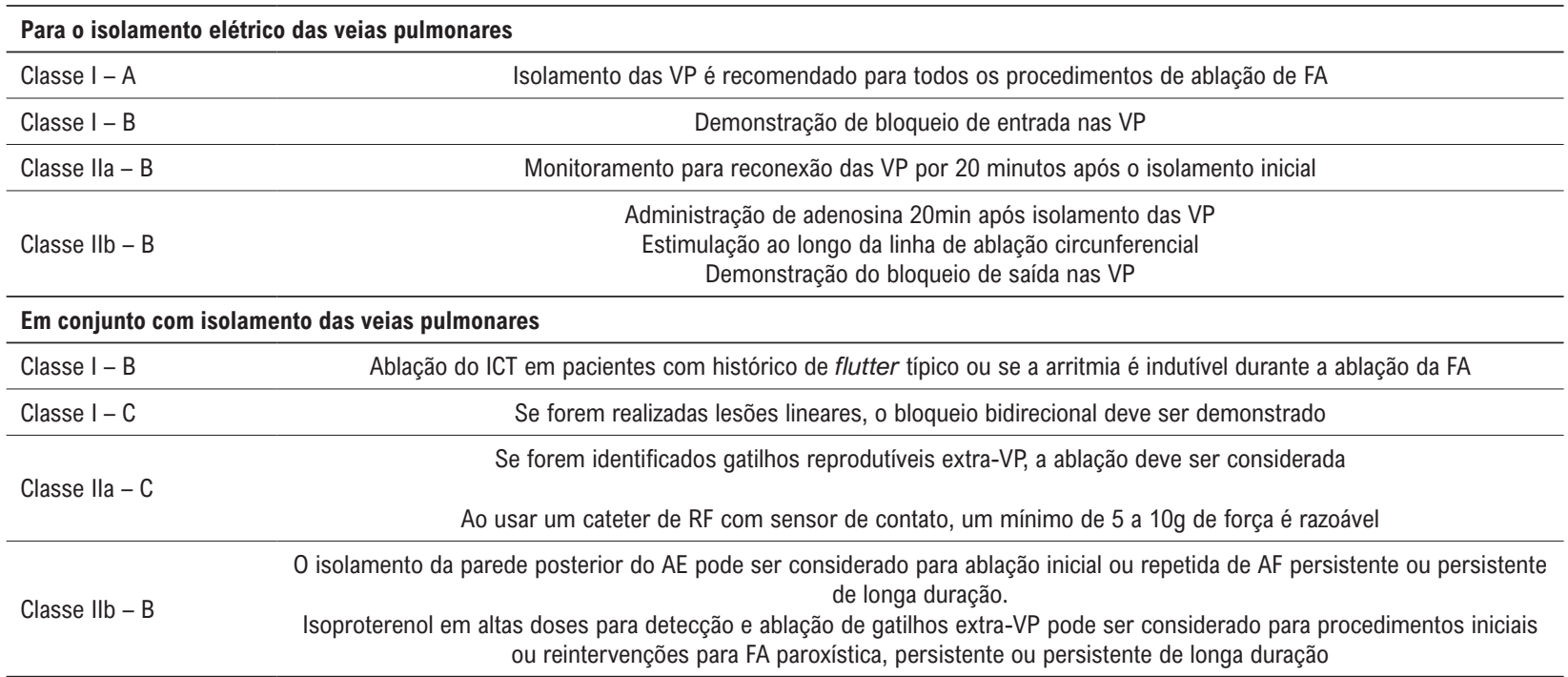

VP: veia pulmonar; FA: fibrilação atrial; RF: radiofrequência; ICT: istmo cavotricuspídeo; AE: átrio esquerdo. 
imagens ultrassonográficas detalhadas da anatomia cardíaca em tempo real, ${ }^{44,45}$ permitindo uma manipulação precisa e segura dos cateteres através das diversas estruturas visualizadas (Figura 2). Sua utilização permite também a realização segura das punções transeptais sob visualização direta e a detecção precoce de complicações agudas (derrame pericárdico, trombos). Estudo recente com mais de 100.000 pacientes submetidos à ablação mostra a importância desse método na significativa redução do risco de uma complicação grave - a perfuração cardíaca. ${ }^{46}$ Nessa série contemporânea, o não uso do ICE foi o maior fator de risco para perfuração (RR 4.85).

Essas ferramentas não fluoroscópicas têm sido cada vez mais utilizadas nos laboratórios de eletrofisiologia ao longo dos anos e podem até orientar todo o procedimento de ablação, evitando completamente o uso de raios X. ${ }^{47}$ Relatado inicialmente há aproximadamente 10 anos, as técnicas "Zero-Fluoro" estão ganhando popularidade na comunidade eletrofisiológica, porque foram demonstradas tão seguras e eficazes quanto os métodos tradicionais guiados pela fluoroscopia. ${ }^{48-50}$

\section{Recorrências}

Dois fatores predominantes justificam as recorrências da FA após ablação:

1. Reconexão da condução nas VP: para que as lesões circunferenciais sejam permanentes, deve ocorrer formação de tecido fibroso, usualmente 4 a 8 semanas após a lesão aguda (edema tecidual). Caso a lesão não tenha profundidade suficiente, pode haver tecido viável remanescente após a reabsorção do edema. Basta um pequeno segmento para restabelecer a condução elétrica.

2. Ocorrência de focos independentes, fora das VP: estes ocorrem mais frequentemente, mas não exclusivamente, em formas persistentes de FA ou em pacientes com remodelamento atrial.
A reconexão das VP é facilmente resolvida com novas aplicações de RF nos gaps de condução. A reintervenção geralmente é rápida, fácil e segura. Em FA paroxística, eleva os índices de controle da FA a 95\%. Com o uso de cateteres com sensor de contato tecidual, é um fenômeno cada vez mais raro, ${ }^{51-53}$ pois as lesões de RF tendem a ser mais profundas e permanentes. ${ }^{54}$

Os focos extra-VP representam um substrato atrial mais difuso, sendo necessários o reconhecimento e a ablação extensa, sem os quais não é possível o controle da arritmia. ${ }^{33,54,55}$ Mais comumente, envolvem a parede posterior do $\mathrm{AE}$, o AAE e o seio coronário ${ }^{32,54}$ - estruturas que podem ser também isoladas por aplicações de RF. É possível a manutenção do ritmo sinusal a longo prazo, mesmo se necessário mais de uma intervenção.

\section{Seleção de pacientes e resultados}

A seleção de pacientes para ablação por cateter da FA baseia-se atualmente na falência do tratamento clínico (Tabela 2). De acordo com o último consenso de especialistas HRS/EHRA/ECAS/APHRS/SOLAECE, de 2017, ${ }^{11}$ a indicação primária para ablação de FA é a presença de fibrilação atrial paroxística ou persistente sintomática, refratária ou intolerante a pelo menos um fármaco antiarrítmico da classe I ou III. Há sólidas evidências de melhora em parâmetros de qualidade de vida desses pacientes. ${ }^{5,56}$

Esta técnica pode ser utilizada em pacientes com diversos tipos de cardiopatia (doença arterial coronária, hipertrofia ventricular esquerda, insuficiência cardíaca) e apresentações clínicas de FA (paroxística, persistente ou de longa duração), porém, os melhores resultados são obtidos nos pacientes com coração estruturalmente normal. No maior estudo randomizado que comparou ablação com tratamento farmacológico (CABANA), ${ }^{7}$ sobrevida livre de recorrência da FA é significativamente melhor (HR 0.53)

Tabela 2 - Indicações para ablação por cateter na fibrilação atrial

\begin{tabular}{|c|c|}
\hline \multicolumn{2}{|c|}{ FA sintomática, refratária ou intolerante a pelo menos 1 fármaco antiarrítmico (classe I ou III) } \\
\hline Classe I-A & FA paroxística \\
\hline Classe lla - B & FA persistente \\
\hline Classe Illb-C & FA persistente de longa duração \\
\hline \multicolumn{2}{|c|}{ FA sintomática, antes do início de fármacos antiarrítmicos (Classe I ou III) } \\
\hline Classe lla - B & FA paroxística \\
\hline Classe lla - C & FA persistente \\
\hline Classe Ilb-C & FA persistente de longa duração \\
\hline \multicolumn{2}{|c|}{ Indicações em populações de pacientes pouco representadas em ensaios clínicos } \\
\hline Classe Ila - B & $\begin{array}{l}\text { Insuficiência cardíaca congestiva } \\
\text { Pacientes } \geq 75 \text { anos de idade } \\
\text { Cardiomiopatia hipertrófica } \\
\text { Jovens ( } \leq 45 \text { anos de idade) } \\
\text { Síndrome taqui-bradi }\end{array}$ \\
\hline Classe lla - C & Atletas com FA \\
\hline Classe IIb - C & FA assintomática \\
\hline
\end{tabular}

FA: fibrilação atrial. 


\section{Artigo de Revisão}

\begin{tabular}{|c|c|c|c|c|c|c|}
\hline Estudo (ano) & Tipo & $\begin{array}{l}\text { Estratégia de } \\
\text { ablação }\end{array}$ & $\mathbf{N}^{0}$ & $\begin{array}{l}\text { Follow-up } \\
\text { (meses) }\end{array}$ & $\begin{array}{c}\text { Manutenção do ritmo } \\
\text { sinusal }\end{array}$ & p-valor \\
\hline \multicolumn{7}{|l|}{ FA paroxística } \\
\hline Thermocool AF (2010) & $\begin{array}{l}\text { Randomizado ablação RF } \\
\text { ou DAA; multicêntrico }\end{array}$ & $\begin{array}{l}\text { IVP } \\
\text { CFAE e linhas } \\
\text { opcionais }\end{array}$ & 167 & 12 & $66 \% / 16 \%$ & $<0,001$ \\
\hline STOP AF (2013) & $\begin{array}{l}\text { Randomizado para } \\
\text { crioablação ou DAA; } \\
\text { multicêntrico }\end{array}$ & IVP & 245 & 12 & $70 \% / 7 \%$ & $<0,001$ \\
\hline SMART AF (2014) & $\begin{array}{l}\text { Não randomizado; } \\
\text { cateter sensor de } \\
\text { contato; multicêntrico }\end{array}$ & $\begin{array}{l}\text { IVP } \\
\text { CFAE e linhas } \\
\text { opcionais }\end{array}$ & 172 & 12 & $72,5 \% / \mathrm{NA}$ & $<0,0001$ \\
\hline TOCCASTAR (2015) & $\begin{array}{l}\text { Randomizado com ou } \\
\text { sem cateter sensor de } \\
\text { contato; multicêntrico }\end{array}$ & $\begin{array}{c}\text { IVP } \\
\text { CFAE, gatilhos e } \\
\text { linhas extra-VP } \\
\text { opcionais }\end{array}$ & 300 & 12 & $67,8 \% / 69,4 \%$ & $0,0073^{*}$ \\
\hline RAAFT-2 (2014) & $\begin{array}{c}\text { Randomizado ablação RF } \\
\text { ou DAA (primeira linha); } \\
\text { multicêntrico }\end{array}$ & $\begin{array}{c}\text { IVP } \\
\text { gatilhos extra-VP } \\
\text { opcionais }\end{array}$ & 127 & 24 & $45 \% / 28 \%$ & 0,02 \\
\hline MANTRA-PAF (2012) & $\begin{array}{c}\text { Randomizado ablação RF } \\
\text { ou DAA (primeira linha); } \\
\text { multicêntrico }\end{array}$ & $\begin{array}{c}\mathrm{PVI}+\text { linha teto } \\
\text { linhas mitral e ICT } \\
\text { opcionais }\end{array}$ & 294 & 24 & $\begin{array}{l}\text { Carga de FA: } \\
13 \% / 19 \%\end{array}$ & - \\
\hline FIRE and ICE (2016) & $\begin{array}{l}\text { Randomizado RF } \\
\text { ou crioablação; } \\
\text { multicêntrico }\end{array}$ & IVP & 762 & 12 & $64,1 \%$ (RF)/65,4\% (crio) & - \\
\hline CIRCA DOSE (2019) & $\begin{array}{l}\text { Randomizado RF ou } \\
\text { crio } 4 \text { min ou crio } 2 \mathrm{~min} \text {; } \\
\text { multicêntrico }\end{array}$ & IVP & 346 & 12 & $\begin{array}{l}53,9 \% \text { (RF)/52,2\% (crio } \\
4 \mathrm{~min}) / 51,7 \% \text { (crio } 2 \mathrm{~min} \text { ) }\end{array}$ & 0,87 \\
\hline \multicolumn{7}{|l|}{ FA persistente } \\
\hline TTOP (2014) & $\begin{array}{c}\text { Randomizado ablação RF } \\
\text { ou DAA / CVE }\end{array}$ & IVP + CFAE & 210 & 6 & $56 \% / 26 \%$ & $<0,001$ \\
\hline SARA (2014) & $\begin{array}{c}\text { Randomizado ablação RF } \\
\text { ou DAA; multicêntrico }\end{array}$ & $\begin{array}{l}\text { IVP } \\
\text { CFAE e linhas } \\
\text { opcionais }\end{array}$ & 146 & 12 & $70 \% / 44 \%$ & 0,002 \\
\hline STAR AF II (2015) & $\begin{array}{l}\text { Randomizado } 3 \\
\text { estratégias de ablação } \\
\text { RF; multicêntrico }\end{array}$ & $\begin{array}{c}\text { IVP; } \\
\text { IVP + CFAE; } \\
\text { IVP + Linhas }\end{array}$ & 589 & 18 & $59 \% / 49 \% / 46 \%$ & 0,15 \\
\hline \multicolumn{7}{|c|}{ FA paroxística ou persistente } \\
\hline CABANA (2019) & $\begin{array}{l}\text { Randomizado ablação RF } \\
\text { ou DAA; multicêntrico }\end{array}$ & $\begin{array}{c}\text { IVP } \\
\text { ablação adicional } \\
\text { opcional }\end{array}$ & 2204 & 48,5 & $69 \% / 50 \%$ & - \\
\hline
\end{tabular}

FA: fibrilação atrial; RF: radiofrequência; DAA: drogas antiarritmicas; IVP: isolamento das veias pulmonares; CFAE: eletrogramas atriais fracionados complexos; CVE: cardioversão elétrica; *não inferioridade.

nos pacientes ablacionados quando comparados aos que permanecem em uso de múltiplos fármacos antiarrítmicos. Apesar disso, nesse estudo, não foi demonstrada redução em desfechos duros combinados (morte, acidente vascular cerebral [AVC], sangramento grave ou parada cardíaca) na análise intention to treat, apesar de haver problemas com grande crossover de pacientes para o grupo ablação (27\%). Nesse estudo, os subgrupos que mais se beneficiaram foram os mais jovens ( $<65$ anos) e os pacientes com insuficiência cardíaca congestiva.

A seleção de pacientes com formas persistente e permanente de FA segue o mesmo raciocínio, porém a decisão deve ser individualizada de acordo com o tamanho do $\mathrm{AE}^{57}$ (que é um importante fator preditor de recorrência) e a duração da FA. FA persistente é uma doença heterogênea, com diferentes graus de fibrose atrial e com influência do sistema nervoso autônomo e outros processos fisiopatológicos ainda mal compreendidos, o que explica os resultados heterogêneos observados com diferentes estratégias de ablação. Essa forma exige uma definição individual do substrato e mecanismos envolvidos. ${ }^{58,59}$

É importante notar que, mesmo com a estratégia de extensas aplicações de RF descritas, o índice de recidivas e a necessidade de novos procedimentos são maiores. 
Na experiência de Natale et al., $60 \%$ dos pacientes mantiveram ritmo sinusal sem fármacos após o primeiro procedimento. ${ }^{54}$ Naqueles que foram submetidos a uma segunda intervenção, $80 \%$ mantiveram ritmo sinusal. A Tabela 3 sumariza alguns dos principais estudos publicados.

A ablação por cateter é menos eficaz em determinados subgrupos de pacientes, ${ }^{60}$ em que ainda é necessário avançar no conhecimento fisiopatológico: átrios dilatados e fibrosados, FA persistente ou de longa duração, cardiomiopatia hipertrófica, infiltrado amiloide, obesidade e apneia do sono.

O acompanhamento a longo prazo de pacientes submetidos à ablação por cateter mostra que há a possibilidade de recidivas tardias, ${ }^{61-63}$ na ordem de $7 \%$ ao ano nos primeiros 5 anos. Deve-se destacar que a estimativa do real sucesso da ablação é dificultada pelas inconsistências e heterogeneidades nas definições de sucesso e recorrências nos diferentes estudos. Como exemplo, a maior parte dos estudos considera recidiva qualquer arritmia atrial com duração maior que 30 segundos, uma definição claramente com pouco significado clínico. Nesse cenário, a carga de FA deve ser mais valorizada nas pesquisas futuras.

Com a tendência ao isolamento permanente das VP, observase mais frequentemente a recorrência por aparecimento de focos extra-VP, que devem ser identificados e ablacionados. ${ }^{32,33,54,64}$ Portanto, é importante manter a vigilância com monitoramento periódico dos pacientes, sendo prudente manter a terapia anticoagulante nos pacientes de mais alto risco que não apresentem contraindicações.

A Tabela 4 resume os cuidados adjuvantes para maximizar a segurança e a eficácia do procedimento.

\section{Situações especiais}

Diretrizes internacionais publicadas em 2016 e 2017 e atualizadas em 2019 e 2020 pelas diferentes sociedades internacionais (SBC/HRS/EHRA/ECAS/APHRS/ACC/AHA/ ESC/EHRA) ${ }^{11-13,15}$ recomendam de forma quase consensual o tratamento ablativo em situações especiais (Tabela 2):

\section{1) Ablação como primeira escolha:}

A crescente segurança e a eficácia permitem que a ablação seja oferecida como terapia de primeira linha para tratamento (antes mesmo do uso de fármacos antiarrítmicos) em algumas situações especiais (atletas, jovens, corações normais) ${ }^{65,66} \mathrm{Em}$ pacientes com FA paroxística ou persistente sintomática é indicação Classe Ila. Situações apropriadas para esta estratégia são pacientes com pausas sintomáticas na reversão (síndrome de taquibradi)67 ou atletas competitivos, que frequentemente tem contraindicação ao uso de fármacos.

\section{2) FA em pacientes com insuficiência cardíaca (IC):}

A IC pode predispor um indivíduo à ocorrência de FA através de vários mecanismos, como o aumento da pressão de enchimento do ventrículo esquerdo ou a dilatação e a fibrose do $\mathrm{AE}$, levando à remodelação estrutural e elétrica do átrio. A FA pode aumentar a mortalidade em pacientes com disfunção ventricular esquerda, ${ }^{68}$ portanto, o tratamento da FA nesse subconjunto de pacientes é de importância crucial, ${ }^{69-73}$ dadas as limitações da amiodarona, único medicamento antiarrítmico disponível para esse subgrupo. As mais recentes diretrizes europeias de 2020 referem indicação Classe Ila, ${ }^{15}$ com base em estudos comparativos com amiodarona (AATAC) ${ }^{69}$ e na publicação de estudos randomizados como o $\mathrm{AMICA}^{74} \mathrm{e}$ CASTLE-AF,75 este último realizado em pacientes com IC grave (Fração de ejeção média 32\%), demonstrando uma redução expressiva na mortalidade ou hospitalização por IC (38\%) e na mortalidade cardiovascular (51\%). Tais achados sem precedentes confirmam o prognóstico negativo da FA nessa população e abrem uma nova fronteira de indicações para ablação em centros com experiência e infraestrutura adequados. Resultados positivos recentes são animadores, com demonstração de melhora da função ventricular e reversão do remodelamento atrial. ${ }^{76}$

\section{3) FA em idosos:}

Há estudos que se concentraram em relatar os resultados da ablação da FA em indivíduos mais velhos. O limite de idade para a definição de idosos variou entre $\geq 70,75$ ou 80 . O número de idosos nesses estudos foi, porém, pequeno, com cinco dos sete estudos inscrevendo menos de 100 pacientes, e os maiores resultados relatados em 261 idosos. Os resultados desses estudos fornecem evidências de que a ablação preenche critérios de segurança e eficácia nessa população, ${ }^{77,78}$ apesar de redução nas taxas de sobrevida livre de FA a cada década de idade (Classe Ila).

\section{4) FA em assintomáticos e redução do risco de AVC:}

Ablação da FA (paroxística ou persistente) em pacientes realmente assintomáticos pode ser considerada, ${ }^{79}$ a despeito das poucas evidências de significativa mudança em desfechos "duros" - em particular no risco de fenômenos tromboembólicos/ AVC. Deve ser realizada por operador experiente e após uma discussão detalhada dos riscos e benefícios da realização do procedimento (Classe IIb). Há sólidas evidências de redução de hospitalizaçõe ${ }^{80} \mathrm{e}$ uso de recursos, com relação custo-benefício favorável..$^{10}$ Nesse cenário, deve-se priorizar pacientes com alta probabilidade de sucesso (jovens, FA paroxística, sem remodelamento atrial significativo).

Diversos estudos retrospectivos observacionais apontam para uma significativa redução do risco tromboembólico em pacientes com escore CHADSVASC $\leq 3$ submetidos à ablação bem-sucedida, ${ }^{81-87}$ muitos deles reportando até desfechos favoráveis em pacientes que interromperam a terapia anticoagulante. Dados do estudo KP-RHYTHM ${ }^{88}$ comprovando que a o risco de $\mathrm{AVC}$ é proporcional à carga de $\mathrm{FA}$ em pacientes paroxísticos, independentemente do escore CHADSVASC, e metánalise de estudos randomizados ${ }^{89}$ sugerindo redução de mortalidade e hospitalizações, são compatíveis com a hipótese de redução de risco após uma ablação bem-sucedida.

É preciso ressaltar, porém, que não há evidência direta de estudos randomizados especificamente desenhados para este objetivo; o estudo $\mathrm{CABANA}^{7}$ não demonstrou redução em desfechos combinados em uma população heterogênea (FA paroxística e persistente) submetida à ablação versus tratamento medicamentoso. O recém-publicado EASTAFNET $4^{90}$ demonstrou significativo benefício em desfechos cardiovasculares com uma estratégia inicial visando ao controle 
do ritmo quando comparado com o controle da frequência cardíaca. Contudo, nesse importante estudo randomizado, apenas $20 \%$ dos pacientes foram tratados com ablação.

Por isso, todas as diretrizes atuais recomendam que o tratamento ablativo não tenha como objetivo a suspensão da terapia anticoagulante, ${ }^{11-14}$ que deve ter sua indicação a partir do risco de base do paciente (usualmente indicada em pacientes com escore CHADSVASC $\geq 2$ ). Todos os pacientes submetidos à ablação devem ficar sob uso de anticoagulantes por período mínimo de 2 meses, independentemente dos fatores de risco, e sua continuação por período indeterminado deve ser individualizada pelo escore de risco.

O estudo OCEAN, ${ }^{91}$ em andamento, compara a manutenção da anticoagulação (rivaroxabana) com o ácido acetilsalicílico em pacientes de risco moderado a severo submetidos à ablação e mantendo ritmo sinusal por pelo menos 1 ano após o procedimento. Os resultados devem ajudar a refinar as indicações de anticoagulação a longo prazo pós-ablação.

\section{Complicações}

O procedimento de ablação está associado a pequenas taxas de complicações em centros de excelência com grande volume e experiência, sendo as mais graves complicações individualmente menores que $1 \%$ nos centros com maior experiência. ${ }^{11}$ A Tabela 5 resume as principais complicações e suas incidências relatadas na literatura.

É importante estar atento a uma complicação tardia (nas primeiras semanas) relacionadas à lesão esofagiana devido à proximidade desse órgão com a parede posterior do $\mathrm{AE}$. Durante a aplicação de energia nessa região, deve-se reduzir a potência e o tempo de aplicação, além do monitoramento da temperatura esofágica luminal (Tabela 4). Uma alternativa já em uso corrente consiste em diversos modos de desvio do trajeto do esôfago, de forma a distanciá-lo do local de aplicação de RF. ${ }^{92-95}$ Há relatos de fístulas atrioesofágicas, com elevado índice de mortalidade ${ }^{96-100}$ Felizmente, essa complicação tem incidência estimada ao redor de $0,04 \%$, mas seu reconhecimento precoce pode ser fundamental para evitar um desfecho fatal..$^{99,101-103}$

\section{Perspectivas futuras}

O uso de alta potência de RF com curta duração (high power short duration) tem sido advogado como um modo de produzir lesões teciduais de melhor qualidade, ${ }^{104,105}$ além de provocar lesões mais largas e de menor profundidade e, portanto, com menor risco de danos colaterais (especialmente ao esôfago). Essa técnica foi associada a menor tempo de aplicações de RF e de instrumentação do átrio esquerdo e baixos índices de complicações, ${ }^{106,107}$ impulsionando novas investigações de cateteres que possam fazer lesões mais permanentes em poucos segundos de aplicação. ${ }^{108}$

Há uma grande expectativa com o desenvolvimento de uma nova modalidade de energia para ablação, denominada "eletroporação". Diferentemente das energias térmicas (RF, crioterapia, laser, ultrassom e micro-ondas), cuja propensão é eliminar todos os tecidos indiscriminadamente, a ablação por campo pulsado ou "eletroporação" é uma modalidade ablativa não térmica, na qual os campos elétricos ultrarrápidos $(<1 \mathrm{~s})$ são aplicados ao tecido-alvo de forma seletiva, desestabilizando as membranas celulares e culminando em morte celular. Isso é possível, pois os tecidos têm diferentes limiares para necrose. Essa tecnologia é usada para tratar tumores sólidos irressecáveis devido à proximidade de vasos sanguíneos ou nervos, dada a resistência a campos elétricos pulsados. ${ }^{109,110}$ Os cardiomiócitos têm um dos limiares de lesão tecidual mais baixos, podendo, por isso, ser aplicados limitando o dano colateral, como o esôfago ${ }^{111}$ e o nervo frênico. ${ }^{112}$

Experiência inicial em pacientes submetidos a isolamento ultrarrápido das VP é bastante promissora, com índices de isolamento permanente nunca antes obtidos (100\%). ${ }^{113}$ Essa tecnologia tem grande potencial para substituir definitivamente a RF e outras energias térmicas para tratamento da FA por cateter.

Tabela 4 - Estratégias adjuvantes à ablação de fibrilação atrial

\begin{tabular}{|c|c|}
\hline \multicolumn{2}{|c|}{ Não diretamente relacionado ao procedimento de ablação } \\
\hline Classe Ila - B & $\begin{array}{c}\text { Perda de peso } \\
\text { Considerar o IMC do paciente na avaliação para procedimento de ablação } \\
\text { Pesquisar sinais e sintomas de apneia do sono } \\
\text { Tratar apneia do sono }\end{array}$ \\
\hline \multirow{3}{*}{ Classe IIb-C } & $\begin{array}{l}\text { Interrupção de DAA antes da ablação para melhorar os resultados a longo } \\
\text { prazo não é clara }\end{array}$ \\
\hline & \\
\hline & $\begin{array}{l}\text { Uso de DAA durante o período de cicatrização (3 meses) após a ablação } \\
\text { para melhorar os resultados não é claro }\end{array}$ \\
\hline \multicolumn{2}{|c|}{ Redução do risco durante o procedimento de ablação } \\
\hline Classe I - B & $\begin{array}{l}\text { Delineamento preciso da anatomia das VP para evitar ablação no seu } \\
\text { interior }\end{array}$ \\
\hline Classe I - C & $\begin{array}{c}\text { Redução da potência aplicada na parede posterior do AE, próximo ao } \\
\text { esôfago }\end{array}$ \\
\hline Classe Ila - C & $\begin{array}{l}\text { Utilização de um cateter com sensor de temperatura no lúmen esofágico } \\
\text { para guiar a titulação da energia aplicada }\end{array}$ \\
\hline
\end{tabular}

DAA: drogas antiarritmicas; IMC: índice de massa corporal; VP: veia pulmonar; AE: átrio esquerdo. 


\begin{tabular}{lcc}
\hline Tabela 5 - Complicações relacionadas à ablação da fibrilação atrial & \\
\hline Complicações & Incidência & Teste diagnóstico \\
\hline Morte & $<0,1 \%$ a $0,4 \%$ & - \\
\hline Estenose/oclusão de artéria coronária & $<0,1 \%$ & Cineangiocoronariografia \\
\hline Fístula atrioesofágica & $0,02 \%$ a $0,11 \%$ & Clínica ou angiografia \\
\hline Embolia aérea & $<1 \%$ & TC/RM \\
\hline Estenose VP & $<1 \%$ & Eco; cateterismo cardíaco \\
\hline Síndrome do AE duro & $<1,5 \%$ & Raio X do tórax; fluoroscopia; Sniff test \\
\hline Paralisia permanente do nervo frênico & $0 \%$ a $0,4 \%$ & TC/RM; angiografia cerebral \\
\hline AVC/AIT & $0 \%$ a $2 \%$ & Ultrassom vascular; TC \\
\hline Complicações vasculares & $0,2 \%$ a $1,5 \%$ & Eco \\
\hline Tamponamento cardíaco & $0,2 \%$ a $5 \%$ & Clínica; ECG; Eco \\
\hline Pericardite & $0 \%$ a $5 \%$ & Endoscopia; deglutição de bário; estudo do \\
\hline Gastroparesia & $0 \%$ a $17 \%$ & esvaziamento gástrico \\
\hline
\end{tabular}

TC: tomografia computadorizada; RM: ressonância magnética; VP: veia pulmonar; AE: átrio esquerdo; AVC: acidente vascular cerebral; AlT: ataque isquêmico transitório; ECG: eletrocardiograma.

O estudo ERADICATE-AF, ${ }^{114}$ recentemente publicado, avaliou o efeito adicional da denervação renal por cateter em 302 pacientes hipertensos submetidos à ablação de FA, randomizados para isolamento das VP simples ou combinado à ablação na artéria renal. Nesse estudo, a adição da denervação resultou em melhor sobrevida livre de FA aos 12 meses (72\% vs. 56\%). Esses achados certamente necessitam ser replicados em um modelo cego (sham procedure) de denervação renal, porém, a modulação do sistema nervoso autônomo é um mecanismo fisiopatológico importante e que deve ser melhor explorado.

\section{Conclusão}

A ablação por cateter é o método mais eficaz para controle do ritmo em pacientes com FA, associada à expressiva redução dos sintomas, da carga de FA e das internações hospitalares, com significativa melhora na qualidade de vida. É associada a baixas taxas de complicações quando realizada em centros experientes. Seu papel na redução de eventos tromboembólicos e na mortalidade ainda necessita

\section{Referências}

1. Haissaguerre M, Jais P, Shah DC, Hocini M, Quiniou G, Garrigue S, et al. Spontaneous initiation of atrial fibrillation by ectopic beats originating in the pulmonary veins. N Engl J Med. 1998; 339(10): 659-66.

2. Kanagaratnam L, Tomassoni G, Schweikert R, Pavia S, Bash D, Beheiry $S$, et al. Empirical pulmonary vein isolation in patients with chronic atrial fibrillation using a three-dimensional nonfluoroscopic mapping system: long-term follow-up. Pacing Clin Electrophysiol. 2001; 24(12): 1774-9.

3. Kanj MH, Wazni OM, Natale A. How to do circular mapping catheterguided pulmonary vein antrum isolation: the Cleveland Clinic approach. Heart Rhythm. 2006; 3(7): 866-9. de comprovação definitiva em futuros estudos randomizados, apesar desta forte tendência nos dados atualmente disponíveis.

\section{Contribuição dos autores}

Concepção e desenho da pesquisa, Obtenção de dados, Análise e interpretação dos dados e Revisão crítica do manuscrito quanto ao conteúdo intelectual importante: Saad EB, d'Avila A; Redação do manuscrito: Saad EB.

\section{Potencial conflito de interesses}

Declaro não haver conflito de interesses pertinentes.

\section{Fontes de financiamento}

O presente estudo não teve fontes de financiamento externas.

\section{Vinculação acadêmica}

Não há vinculação deste estudo a programas de pósgraduação.

4. Verma A, Marrouche NF, Natale A. Pulmonary vein antrum isolation: intracardiac echocardiography-guided technique. J CardiovasC Electrophysio.I 2004; 15(11): 1335-40.

5. Mark DB, Anstrom KJ, Sheng S, Piccini JP, Baloch KN, Monahan KH, et al. Effect of Catheter Ablation vs Medical Therapy on Quality of Life Among Patients With Atrial Fibrillation: The CABANA Randomized Clinical Trial. JAMA. 2019; 321(13): 1275-85.

6. Packer DL, Kowal RC, Wheelan KR, Irwin JM, Champagne J, Guerra PG, et al. Cryoballoon ablation of pulmonary veins for paroxysmal atrial fibrillation: first results of the North American Arctic Front (STOP AF) pivotal trial. J Am Coll Cardio.l 2013; 61(16): 1713-23. 
7. Packer DL, Mark DB, Robb RA, Monahan KH, Bahnson TD, PooleJE, et al. Effect of Catheter Ablation vs Antiarrhythmic Drug Therapy on Mortality, Stroke, Bleeding, and Cardiac Arrest Among Patients With Atrial Fibrillation: The CABANA Randomized Clinical Trial. JAMA. 2019; 321(13): 1261-74.

8. Reynolds MR, Walczak J, White SA, Cohen DJ, Wilber DJ. Improvements in symptoms and quality of life in patients with paroxysmal atrial fibrillation treated with radiofrequency catheter ablation versus antiarrhythmic drugs. Circ Cardiovasc Qual Outcomes. 2010;3(6): 615-23.

9. Wilber DJ, Pappone C, Neuzil P, Paola A, Marchlinski F, Natale A, et al. Comparison of antiarrhythmic drug therapy and radiofrequency catheter ablation in patients with paroxysmal atrial fibrillation: a randomized controlled trial. JAMA. 2010; 303(4): 333-40.

10. Saad EB, Tayar DO, Ribeiro RA, Junqueira SM, Jr., Andrade P, d'Avila A. Healthcare Utilization and Costs Reduction after Radiofrequency Ablation For Atrial Fibrillation in the Brazilian Private Healthcare System. Arq Bras Cardiol. 2019; 113(2): 252-7.

11. Calkins H, Hindricks G, Cappato R, Kim YH, Saad EB, Aguinaga L, et al. 2017 HRS/EHRA/ECAS/APHRS/SOLAECE expert consensus statement on catheter and surgical ablation of atrial fibrillation. Heart Rhythm. 2017;14(10): e275-e444.

12. January CT, Wann LS, Calkins H, Chen LY, Cigarroa JE, Cleveland Jr JC, et al. 2019 AHA/ACC/HRS Focused Update of the 2014 AHA/ACC/HRS Guideline for the Management of Patients With Atrial Fibrillation: A Report of the American College of Cardiology/American Heart Association Task Force on Clinical Practice Guidelines and the Heart Rhythm Society in Collaboration With the Society of Thoracic Surgeons. Circulation. 2019; 140(2):e125-e51.

13. Kirchhof P, Benussi S, Kotecha D, Ahlsson A, Atar D, Casadei B, etal. 2016 ESC Guidelines for the management of atrial fibrillation developed in collaboration with EACTS. Eur Heart J. 2016; 37(38): 2893-962.

14. Magalhaes LP, Figueiredo MJO, Cintra FD, Saad EB, Kuniyoshi RR, Lorga Filho AM, et al. Executive Summary of the II Brazilian Guidelines for Atrial Fibrillation. Arq Bras Cardiol 2016; 107(6): 501-8.

15. Hindricks G, Potpara T, Dagres N, Arbelo E, Bax JJ, Blomström-Lundqvist C, et al. 2020 ESC Guidelines for the diagnosis and management of atrial fibrillation developed in collaboration with the European Association of Cardio-Thoracic Surgery (EACTS). Eur Heart). 2020.

16. Silva MA, Futuro GMC, Mercon ES, Vasconcelos D, Agrizzi RS, Elias Neto J, et al. Safety of Catheter Ablation of Atrial Fibrillation Under Uninterrupted Rivaroxaban Use. Arq Bras Cardiol. 2020; 114(3): 435-42.

17. Saad EB, Costa IP, Costa RE, Inacio Jr LA, Slater C, Camiletti A, et al. Safety of ablation for atrial fibrillation with therapeutic INR: comparison with transition to low-molecular-weight heparin. Arq Bras Cardiol. 2011; 97(4): 289-96.

18. Calkins $\mathrm{H}$, Willems $\mathrm{S}$, Gerstenfeld EP, Verma A, Schilling R, Hohnloser $\mathrm{SH}$, et al. Uninterrupted Dabigatran versus Warfarin for Ablation in Atrial Fibrillation. N Engl J Med. 2017; 376(17):1627-36.

19. Arruda M, Mlcochova H, Prasad SK, Kilicaslan F, Saliba W, Patel D, et al. Electrical isolation of the superior vena cava: an adjunctive strategy to pulmonary vein antrum isolation improving the outcome of $\mathrm{AF}$ ablation. J Cardiovasc Electrophysiol. 2007; 18(12):1261-6.

20. Gianni C, Sanchez JE, Mohanty S, Trivedi C, Rocca DG, Al-Ahmad A, et al. Isolation of the superior vena cava from the right atrial posterior wall: a novel ablation approach. Europace. 2018; 20(9): e124-e32.

21. Kuck KH, Brugada J, Furnkranz A, Metzner A, Ouyang F, Chun KR, et al. Cryoballoon or Radiofrequency Ablation for Paroxysmal Atrial Fibrillation. N EnglJ Med. 2016; 374(23):2235-45.

22. Andrade JG, Champagne J, Dubuc M, Deyell MW, Verma A, Macle L, et al. Cryoballoon or Radiofrequency Ablation for Atrial Fibrillation Assessed by Continuous Monitoring: A Randomized Clinical Trial. Circulation. 2019; 140(22):1779-88.

23. Oral H, Knight BP, Tada H, Ozaydin M, Chugh A, Hassan S, et al. Pulmonary vein isolation for paroxysmal and persistent atrial fibrillation. Circulation. 2002;105(9):1077-81.
24. Parkash R, Tang AS, Sapp JL, Wells G. Approach to the catheter ablation technique of paroxysmal and persistent atrial fibrillation: a meta-analysis of the randomized controlled trials. J Cardiovasc Electrophysiol. 2011; 22(7):729-38

25. Wynn GJ, Das M, Bonnett LJ, Panikker S, Wong T, Gupta D. Efficacy of catheter ablation for persistent atrial fibrillation: a systematic review and meta-analysis of evidence from randomized and nonrandomized controlled trials. Circ Arrhythm Electrophysiol. 2014; 7(5):841-52.

26. Scott PA, Silberbauer J, Murgatroyd FD. The impact of adjunctive complex fractionated atrial electrogram ablation and linear lesions on outcomes in persistent atrial fibrillation: a meta-analysis. Europace. 2016; 18(3): 359-67.

27. Romero J, Gianni C, Natale A, Di Biase L. What Is the Appropriate Lesion Set for Ablation in Patients with Persistent Atrial Fibrillation? Curr Treat Options Cardiovasc. Med 2017; 19(5): 35.

28. Mohanty S, Mohanty P, Trivedi C, Gianni C, Rocca DG, Biasi L, et al. Long-Term Outcome of Pulmonary Vein Isolation With and Without Focal Impulse and Rotor Modulation Mapping: Insights From a Meta-Analysis. Circ Arrhythm Electrophysiol. 2018; 11(3): e005789.

29. Hung Y, Lo LW, Lin YJ, Chang SL, Hu YF, Chung FP, et al. Characteristics and long-term catheter ablation outcome in long-standing persistent atrial fibrillation patients with non-pulmonary vein triggers. Int J Cardiol. 2017; 241: 205-11.

30. Bai R, Di Biase L, Mohanty P, Trivedi C, Russo AD, Themistoclakis S, et al. Proven isolation of the pulmonary vein antrum with or without left atrial posterior wall isolation in patients with persistent atrial fibrillation. Heart Rhythm. 2016; 13(1): 132-40.

31. Pambrun T, Denis A, Duchateau J, Sacher F, Hocini M, Jais P, etal. MARSHALL bundles elimination, Pulmonary veins isolation and Lines completion for ANatomical ablation of persistent atrial fibrillation: MARSHALL-PLAN case series. J Cardiovasc Electrophysiol. 2019; 30(1): 7-15.

32. Santangeli P, Zado ES, Hutchinson MD, Riley MP, Lin D, Frankel DS, et al. Prevalence and distribution of focal triggers in persistent and long-standing persistent atrial fibrillation. Heart Rhythm. 2016; 13(2): 374-82.

33. Hayashi K, An Y, Nagashima M, Hiroshima K, Ohe M, Makihara Y, et al. Importance of nonpulmonary vein foci in catheter ablation for paroxysmal atrial fibrillation. Heart Rhythm. 2015; 12(9):1918-24.

34. Kottkamp H, Berg J, Bender R, Rieger A, Schreiber D. Box Isolation of Fibrotic Areas (BIFA): A Patient-Tailored Substrate Modification Approach for Ablation of Atrial Fibrillation. J Cardiovasc Electrophysiol. 2016; 27(1): 22-30.

35. Thiyagarajah A, Kadhim K, Lau DH, Emami M, Linz D, Khokhar K, et al. Feasibility, Safety, and Efficacy of Posterior Wall Isolation During Atrial Fibrillation Ablation: A Systematic Review and Meta-Analysis. Circ Arrhythm Electrophysiol. 2019; 12(8):e007005.

36. Di Biase L, Burkhardt JD, Mohanty P, Sanchez J, Mohanty S, Horton R, et al. Left atrial appendage: an underrecognized trigger site of atrial fibrillation. Circulation. 2010; 122(2):109-18.

37. Brooks S, Metzner A, Wohlmuth P, Lin T, Wissner E, Tilz R, et al. Insights into ablation of persistent atrial fibrillation: Lessons from 6-year clinical outcomes. J Cardiovasc Electrophysiol. 2018; 29(2): 257-63.

38. Verma A, Jiang CY, Betts TR, Chen J, Deisenhofer I, Mantovan R, et al. Approaches to catheter ablation for persistent atrial fibrillation. N Engl J Med. 2015;372(19):1812-22.

39. Di Biase L, Burkhardt JD, Mohanty P, Mohanty S, Sanches JE, Trivedi C, et al. Left Atrial Appendage Isolation in Patients With Longstanding Persistent AF Undergoing Catheter Ablation: BELIEF Trial. J Am Coll Cardiol. 2016; 68(18):1929-40.

40. Di Biase L, Mohanty S, Trivedi C, Romero J, Natale V, Briceno D, et al. Stroke Risk in Patients With Atrial Fibrillation Undergoing Electrical Isolation of the Left Atrial Appendage. J Am Coll Cardiol. 2019; 74(8): 1019-28. 
41. Marrouche NF, Wilber D, Hindricks G, Jais P, Akoum N, Marchlinsk F, et al. Association of atrial tissue fibrosis identified by delayed enhancement MRI and atrial fibrillation catheter ablation: the DECAAF study. JAMA. 2014; 311(5):498-506.

42. Goette A, Kalman JM, Aguinaga L, Akar J, Cabrera JA, Chen AS, et al. EHRA/HRS/APHRS/SOLAECE expert consensus on atria cardiomyopathies: Definition, characterization, and clinical implication. Heart Rhythm. 2017; 14(1): e3-e40.

43. Bunch TJ, May HT, Bair TL, Johnson DL, Weiss JP, Crandall BG, et al. Increasing time between first diagnosis of atrial fibrillation and catheter ablation adversely affects long-term outcomes. Heart Rhythm. 2013; 10(9): 1257-62.

44. Enriquez A, Saenz LC, Rosso R, Silvestry FE, Callans D, Marchlinski FE, et al. Use of Intracardiac Echocardiography in Interventional Cardiology: Working With the Anatomy Rather Than Fighting It. Circulation. 2018; 137(21): 2278-94.

45. Saad EB, Costa IP, Camanho LE. Use of intracardiac echocardiography in the electrophysiology laboratory. Arq Bras Cardiol. 2011; 96(1): e11-7.

46. Friedman DJ, Pokorney SD, Ghanem A, Marcello S, Kalsekar I, Yadalam $\mathrm{S}$, et al. Predictors of Cardiac Perforation With Catheter Ablation of Atrial Fibrillation. JACC Clin Electrophysiol. 2020; 6(6): 636-45.

47. Heidbuchel H, Wittkampf FH, Vano E, Ernst S, Schilling R, Picano E, et al. Practical ways to reduce radiation dose for patients and staff during device implantations and electrophysiological procedures. Europace. 2014; 16(7): 946-64.

48. Lerman BB, Markowitz SM, Liu CF, Thomas G, Ip JE, Cheung JW. Fluoroless catheter ablation of atrial fibrillation. Heart Rhythm. 2017; 14(6): 928-34.

49. Razminia M, Willoughby MC, Demo H, Keshmiri H, Wang T, D'Silva O, et al. Fluoroless Catheter Ablation of Cardiac Arrhythmias: A 5-Year Experience. Pacing Clin Electrophysiol. 2017; 40(4): 425-33.

50. Saad EB, Slater C, Inacio LAO, Jr., Santos GVD, Dias LC, Camanho LEM. Catheter Ablation for Treatment of Atrial Fibrillation and Supraventricular Arrhythmias Without Fluoroscopy Use: Acute Efficacy and Safety. Arq Bras Cardiol. 2020; 114(6): 1015-26.

51. Natale A, Reddy VY, Monir G, Wilber DJ, Lindsay BD, McElderry HT, et al. Paroxysmal AF catheter ablation with a contact force sensing catheter: results of the prospective, multicenter SMART-AF trial. J Am Coll Cardiol. 2014; 64(7): 647-56.

52. Reddy VY, Dukkipati SR, Neuzil P, Natale A, Albenque AP, Kautzner J, et al. Randomized, Controlled Trial of the Safety and Effectiveness of a Contact Force-Sensing Irrigated Catheter for Ablation of Paroxysmal Atrial Fibrillation: Results of the TactiCath Contact Force Ablation Catheter Study for Atrial Fibrillation (TOCCASTAR) Study. Circulation. 2015; 132(10): 907-15.

53. De Pooter J, Strisciuglio T, El Haddad M, Wolf M, Phlips T, Vandekerckhove $Y$, et al. Pulmonary Vein Reconnection No Longer Occurs in the Majority of Patients After a Single Pulmonary Vein Isolation Procedure. JACC Clin Electrophysiol. 2019; 5(3): 295-305

54. Gokoglan Y, Mohanty S, Gunes MF, Trivedi C, Santangeli P, Gianni C, et al. Pulmonary Vein Antrum Isolation in Patients With Paroxysmal Atrial Fibrillation: More Than a Decade of Follow-Up. Circ Arrhythm Electrophysiol. 2016; 9(5)e003660.

55. Della Rocca DG, Mohanty S, Trivedi C, Di Biase L, Natale A. Percutaneous Treatment of Non-paroxysmal Atrial Fibrillation: A Paradigm Shift from Pulmonary Vein to Non-pulmonary Vein Trigger Ablation? Arrhythm Electrophysiol Rev. 2018; 7(4): 256-60.

56. Blomstrom-Lundqvist C, Gizurarson S, Schwieler J, Jensen SM, Bergfeldt L, Kenneback G, et al. Effect of Catheter Ablation vs Antiarrhythmic Medication on Quality of Life in Patients With Atrial Fibrillation: The CAPTAF Randomized Clinical Trial. JAMA. 2019; 321(11): 1059-68.

57. Njoku A, Kannabhiran M, Arora R, Reddy P, Gopinathannair R, Lakkireddy $D$, et al. Left atrial volume predicts atrial fibrillation recurrence after radiofrequency ablation: a meta-analysis. Europace. 2018; 20(1): 33-42.
58. Mohanty S, Mohanty P, Di Biase L, Trivedi C, Morris EH, Gianni C, et al Long-term follow-up of patients with paroxysmal atrial fibrillation and severe left atrial scarring: comparison between pulmonary vein antrum isolation only or pulmonary vein isolation combined with either scar homogenization or trigger ablation. Europace. 2017; 19(11): 1790-7.

59. Fochler F, Yamaguchi T, Kheirkahan M, Kholmovski EG, Morris AK, Marrouche NF. Late Gadolinium Enhancement Magnetic Resonance Imaging Guided Treatment of Post-Atrial Fibrillation Ablation Recurrent Arrhythmia. Circ Arrhythm Electrophysiol. 2019; 12(8): e007174.

60. Al-Khatib SM, Benjamin EJ, Buxton AE, Calkins H, Chung MK, Curtis $A B$, et al. Research Needs and Priorities for Catheter Ablation of Atrial Fibrillation: A Report From a National Heart, Lung, and Blood Institute Virtual Workshop. Circulation. 2020; 141(6): 482-92.

61. Steinberg JS, Palekar R, Sichrovsky T, Arshad A, Preminger M, Musat D, et al. Very long-term outcome after initially successful catheter ablation of atrial fibrillation. Heart Rhythm. 2014; 11(5): 771-6.

62. Takigawa M, Takahashi A, Kuwahara T, Okubo K, Takahashi Y, Watari $Y$, et al. Long-term follow-up after catheter ablation of paroxysmal atrial fibrillation: the incidence of recurrence and progression of atrial fibrillation. Circ Arrhythm Electrophysiol. 2014; 7(2): 267-73.

63. Tilz RR, Heeger CH, Wick A, Saguner AM, Metzner A, Rillig A, et al. TenYear Clinical Outcome After Circumferential Pulmonary Vein Isolation Utilizing the Hamburg Approach in Patients With Symptomatic DrugRefractory Paroxysmal Atrial Fibrillation. Circ Arrhythm Electrophysiol. 2018; $11(2)$ : e005250.

64. Lin D, Santangeli P, Zado ES, Bala R, Hutchinson MD, Riley MP, et al. Electrophysiologic findings and long-term outcomes in patients undergoing third or more catheter ablation procedures for atrial fibrillation. $J$ Cardiovasc Electrophysiol. 2015; 26(4): 371-7.

65. Morillo CA, Verma A, Connolly SJ, Kuck KH, Nair GM, Champagne J, et al. Radiofrequency ablation vs antiarrhythmic drugs as first-line treatment of paroxysmal atrial fibrillation (RAAFT-2): a randomized trial. JAMA. 2014; 311(7): 692-700.

66. Walfridsson $\mathrm{H}$, Walfridsson $\mathrm{U}$, Nielsen $\mathrm{JC}$, Johannessen $\mathrm{A}$, Raatikainen $\mathrm{P}$ Janzon $\mathrm{M}$, et al. Radiofrequency ablation as initial therapy in paroxysmal atrial fibrillation: results on health-related quality of life and symptom burden. The MANTRA-PAF trial. Europace. 2015; 17(2): 215-21.

67. Khaykin Y, Marrouche NF, Martin DO, Saliba W, Schweikert R, Wexman $M$, et al. Pulmonary vein isolation for atrial fibrillation in patients with symptomatic sinus bradycardia or pauses. J Cardiovasc Electrophysiol. 2004; 15(7): 784-9.

68. Swedberg K, Olsson LG, Charlesworth A, Cleland J, Hanrath P, Komajda M, et al. Prognostic relevance of atrial fibrillation in patients with chronic heart failure on long-term treatment with beta-blockers: results from COMET. Eur Heart J. 2005; 26(13): 1303-8.

69. Di Biase L, Mohanty P, Mohanty S, Santangeli P, Trivedi C, LakkiReddy D, et al. Ablation Versus Amiodarone for Treatment of Persistent Atrial Fibrillation in Patients With Congestive Heart Failure and an Implanted Device: Results From the AATAC Multicenter Randomized Trial. Circulation. 2016; 133(17): 1637-44

70. Hunter RJ, Berriman TJ, Diab I, Kamdar R, Richmond L, Baker V, et al. A randomized controlled trial of catheter ablation versus medical treatment of atrial fibrillation in heart failure (the CAMTAF trial). Circ Arrhythm Electrophysiol. 2014; 7(1): 31-8.

71. Roy D, Talajic M, Nattel S, Wyse DG, Dorian P, Lee KL, et al. Rhythm control versus rate control for atrial fibrillation and heart failure. N Eng/J Med. 2008; 358(25): 2667-77.

72. Lobo TJ, Pachon CT, Pachon JC, Pachon El, Pachon MZ, Pachon JC et al. Atrial fibrillation ablation in systolic dysfunction: clinical and echocardiographic outcomes. Arq Bras Cardiol. 2015; 104(1): 45-52.

73. Scanavacca M, Bocchi EA. Catheter Ablation for Atrial Fibrillation in Patients with Heart Failure. Arq Bras Cardiol. 2018; 110(4): 300-2. 
74. Kuck KH, Merkely B, Zahn R, Arentz T, Seidl K, Schluter M, et al. Catheter Ablation Versus Best Medical Therapy in Patients With Persistent Atrial Fibrillation and Congestive Heart Failure: The Randomized AMICA Trial. Circ Arrhythm Electrophysiol. 2019; 12(12): e007731.

75. Marrouche NF, Brachmann J, Andresen D, Siebels J, Boersma L, Jordaens L, et al. Catheter Ablation for Atrial Fibrillation with Heart Failure. N Engl J Med. 2018; 378(5): 417-27.

76. Sugumar H, Prabhu S, Voskoboinik A, Young S, Gutman SJ, Wong JR, et al. Atrial Remodeling Following Catheter Ablation for Atrial FibrillationMediated Cardiomyopathy: Long-Term Follow-Up of CAMERA-MRI Study. JACC Clin Electrophysiol. 2019; 5(6): 681-8.

77. Nademanee K, Amnueypol M, Lee F, Drew CM, Suwannasri W, Schwab $\mathrm{MC}$, et al. Benefits and risks of catheter ablation in elderly patients with atrial fibrillation. Heart Rhythm. 2015; 12(1): 44-51.

78. Santangeli P, Di Biase L, Mohanty P, Burkhardt JD, Horton R, Bai R, et al. Catheter ablation of atrial fibrillation in octogenarians: safety and outcomes. J Cardiovasc Electrophysiol. 2012; 23(7): 687-93.

79. Kalman JM, Sanders P, Rosso R, Calkins H. Should We Perform Catheter Ablation for Asymptomatic Atrial Fibrillation? Circulation. 2017; 136(5): 490-9.

80. Guo J, Nayak HM, Besser SA, Beaser A, Aziz Z, Broman M, et al. Impact of Atrial Fibrillation Ablation on Recurrent Hospitalization: A Nationwide Cohort Study. JACC Clin Electrophysiol. 2019; 5(3): 330-9.

81. Saad EB, d'Avila A, Costa IP, Aryana A, Slater C, Costa RE, et al. Very low risk of thromboembolic events in patients undergoing successful catheter ablation of atrial fibrillation with a CHADS2 score $\leq 3$ : a long-term outcome study. Circ Arrhythm Electrophysiol. 2011; 4(5): 615-21.

82. Proietti R, AlTurki A, Di Biase L, China P, Forleo G, Corrado A, et al. Anticoagulation after catheter ablation of atrial fibrillation: An unnecessary evil? A systematic review and meta-analysis. J Cardiovasc Electrophysiol. 2019; 30(4): 468-78.

83. Themistoclakis S, Corrado A, Marchlinski FE, Jais P, Zado E, Rossillo A, et al. The risk of thromboembolism and need for oral anticoagulation after successful atrial fibrillation ablation. J Am Coll Cardiol. 2010; 55(8): 73543.

84. Bunch TJ, May HT, Bair TL, Weiss JP, Crandall BG, Osborn JS, et al. Atrial fibrillation ablation patients have long-term stroke rates similar to patients without atrial fibrillation regardless of CHADS2 score. Heart Rhythm. 2013; 10(9): 1272-7.

85. Bunch TJ, Crandall BG, Weiss JP, May HT, Bair TL, Osborn JS, et al. Patients treated with catheter ablation for atrial fibrillation have long-term rates of death, stroke, and dementia similar to patients without atrial fibrillation. J Cardiovasc Electrophysiol. 2011; 22(8): 839-45.

86. Kalbfleisch SJ. Atrial fibrillation ablation, stroke, and mortality: Evaluating the effects of therapy in the era of big data. Heart Rhythm. 2017; 14(5): $643-4$

87. Saliba W, Schliamser JE, Lavi I, Barnett-Griness O, Gronich N, Rennert G. Catheter ablation of atrial fibrillation is associated with reduced risk of stroke and mortality: A propensity score-matched analysis. Heart Rhythm. 2017; 14(5): 635-42.

88. Go AS, Reynolds K, Yang J, Gupta N, Lenane J, Sung SH, et al. Association of Burden of Atrial Fibrillation With Risk of Ischemic Stroke in Adults With Paroxysmal Atrial Fibrillation: The KP-RHYTHM Study. JAMA Cardiol. $2018 ; 3(7): 601-8$.

89. Asad ZUA, Yousif A, Khan MS, Al-Khatib SM, Stavrakis S. Catheter Ablation Versus Medical Therapy for Atrial Fibrillation: A Systematic Review and Meta-Analysis of Randomized Controlled Trials. Circ Arrhythm Electrophysiol. 2019; 12(9): e007414.

90. Kirchhof P, Camm AJ, Goette A, Brandes A, Eckardt L, Elvan A, et al. Early Rhythm-Control Therapy in Patients with Atrial Fibrillation. N Engl J Med. 2020 Oct 1;383(14):1305-1316.
91. Verma A, Ha ACT, Kirchhof P, Hindricks G, Healey JF, Hill MD, et al. The Optimal Anti-Coagulation for Enhanced-Risk Patients Post-Catheter Ablation for Atrial Fibrillation (OCEAN) trial. Am Heart J. 2018; 197: 124-32.

92. Mateos JC, Mateos EI, Pena TG, Lobo TJ, Mateos JC, Vargas RN, et al. Simplified method for esophagus protection during radiofrequency catheter ablation of atrial fibrillation--prospective study of 704 cases. Rev Bras Cir Cardiovasc. 2015; 30(2): 139-47.

93. Bhardwaj R, Naniwadekar A, Whang W, Mittnacht AJ, Palaniswamy C, Koruth JS, et al. Esophageal Deviation During Atrial Fibrillation Ablation: Clinical Experience With a Dedicated Esophageal Balloon Retractor. JACC Clin Electrophysiol. 2018; 4(8): 1020-30.

94. Iwasawa J, Koruth JS, Mittnacht AJ, Tran VN, Palaniswamy C, Sharma D, et al. The impact of mechanical oesophageal deviation on posterior wall pulmonary vein reconnection. Europace. 2020; 22(2): 232-9.

95. Palaniswamy C, Koruth JS, Mittnacht AJ, Miller MA, Choudry S, Bhardwaj $R$, et al. The Extent of Mechanical Esophageal Deviation to Avoid Esophageal Heating During Catheter Ablation of Atrial Fibrillation. JACC Clin Electrophysiol. 2017; 3(10): 1146-54.

96. Vasconcelos JT, Filho S, Atie J, Maciel W, Souza OF, Saad EB, et al. Atrialoesophageal fistula following percutaneous radiofrequency catheter ablation of atrial fibrillation: the risk still persists. Europace. 2017; 19(2): 250-8.

97. Calkins H, Natale A, Gomez T, Etlin A, Bishara M. Comparing rates of atrioesophageal fistula with contact force-sensing and non-contact forcesensing catheters: analysis of post-market safety surveillance data. J Interv Card Electrophysiol. 2019.

98. Gunes MF, Gokoglan Y, Biase L, Gianni C, Mohanty S, Horton R, et al. Ablating the Posterior Heart: Cardioesophageal Fistula Complicating Radiofrequency Ablation in the Coronary Sinus. J Cardiovasc Electrophysiol. 2015; 26(12): 1376-8.

99. Mohanty S, Santangeli P, Mohanty P, Biase L, Trivedi C, Bai R, et al. Outcomes of atrioesophageal fistula following catheter ablation of atrial fibrillation treated with surgical repair versus esophageal stenting. J Cardiovasc Electrophysiol. 2014; 25(6): 579-84.

100. Han HC, Ha FJ, Sanders P, Spencer R, Teh AW, O'Donnell D, et al. Atrioesophageal Fistula: Clinical Presentation, Procedural Characteristics, Diagnostic Investigations, and Treatment Outcomes. Circ Arrhythm Electrophysiol. 2017; 10(11).

101. Wu TC, Pisani C, Scanavacca MI. Approaches to the Diagnosis and Management of Atrial-Esophageal Fistula After Catheter Ablation for Atrial Arrhythmias. Curr Cardiovasc Risk Rep. 2019; 13.

102. Pappone C, Vicedomini G, Santinelli V. Atrio-Esophageal Fistula After AF Ablation: Pathophysiology, Prevention \& Treatment. J Atr Fibrillation. 2013; 6(3): 860 .

103. Kim YG, Shim J, Lee KN, Lim JY, Chung JH, Jung JS, et al. Management of Atrio-esophageal Fistula Induced by Radiofrequency Catheter Ablation in Atrial Fibrillation Patients: a Case Series. Sci Rep. 2020; 10(1): 8202.

104. Bhaskaran A, Chik W, Pouliopoulos J, Nalliah C, Qian P, Barry T, et al. Five seconds of 50-60 $\mathrm{W}$ radio frequency atrial ablations were transmural and safe: an in vitro mechanistic assessment and force-controlled in vivo validation. Europace. 2017; 19(5): 874-80.

105. Leshem E, Zilberman I, Tschabrunn CM, Barkagan M, Contreras-Valdes FM, Govari A, et al. High-Power and Short-Duration Ablation for Pulmonary Vein Isolation: Biophysical Characterization. JACC Clin Electrophysiol. 2018; 4(4): 467-79.

106. Winkle RA, Moskovitz R, Hardwin Mead R, Engel G, Kong MH, Fleming $\mathrm{W}$, et al. Atrial fibrillation ablation using very short duration $50 \mathrm{~W}$ ablations and contact force sensing catheters. Interv Card Electrophysiol. 2018; 52(1): 1-8.

107. Winkle RA, Mohanty S, Patrawala RA, Mead RH, Kong MH, Engel G, et al. Low complication rates using high power (45-50 W) for short duration for atrial fibrillation ablations. Heart Rhythm. 2019; 16(2): 165-9. 
108. Reddy VY, Grimaldi M, De Potter T, Vijgen JM, Bulava A, Duytschaever MF, et al. Pulmonary Vein Isolation With Very High Power, Short Duration, Temperature-Controlled Lesions: The QDOT-FAST Trial. JACC Clin Electrophysiol. 2019; 5(7): 778-86.

109. Rubinsky B, Onik G, Mikus P. Irreversible electroporation: a new ablation modality--clinical implications. Technol Cancer Res Treat. 2007; 6(1): 37-48.

110. Davalos RV, Mir IL, Rubinsky B. Tissue ablation with irreversible electroporation. Ann Biomed Eng. 2005; 33(2): 223-31.

111. Koruth JS, Kuroki K, Kawamura I, Brose R, Viswanathan R, Buck ED, et al. Pulsed Field Ablation vs Radiofrequency Ablation: Esophageal Injury in a Novel Porcine Model. Circ Arrhythm Electrophysiol. 2020 Mar;13(3):e008303.
112. Koruth JS, Kuroki K, Iwasawa J, Viswanathan R, Brose R, Buck ED, et al Endocardial ventricular pulsed field ablation: a proof-of-concept preclinical evaluation. Europace. 2020 Mar 1;22(3):434-9

113. Reddy VY, Neuzil P, Koruth JS, Petru J, Funosako M, Cochet H, et al. Pulsed Field Ablation for Pulmonary Vein Isolation in Atrial Fibrillation. J Am Coll Cardiol. 2019; 74(3): 315-26.

114. Steinberg JS, Shabanov V, Ponomarev D, Losik D, Ivanickiy E, Kropotkin E, et al. Effect of Renal Denervation and Catheter Ablation vs Catheter Ablation Alone on Atrial Fibrillation Recurrence Among Patients With Paroxysmal Atrial Fibrillation and Hypertension: The ERADICATE-AF Randomized Clinical Trial. JAMA. 2020; 323(3): 248-55. 